\title{
Aceleração do crescimento em diâmetro de espécies da Floresta Ombrófila Mista nos últimos 90 anos
}

\author{
Patricia Póvoa de Mattos ${ }^{1}$, Mariana Ferraz Oliveira², Andrea Fernanda Agustini², Evaldo Muñoz Braz¹, Hugo \\ Rivera $^{3}$, Yeda Maria Malheiros de Oliveira ${ }^{1}$, Maria Augusta Doetzer Rosot ${ }^{1}$, Marilice Cordeiro Garrastazu ${ }^{1}$ \\ ${ }^{1}$ Embrapa Florestas, Estrada da Ribeira, Km 111, CP 319, CEP 83411-000, Colombo, PR, Brasil, povoa@cnpf.embrapa.br;

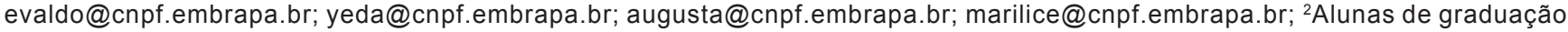 \\ do curso de Engenharia Florestal, Universidade Federal do Paraná, mariana.ferraz@gmail.com, deia_agustini@hotmail.com; \\ ${ }^{3}$ Corporación Nacional Forestal (Conaf-Chile), Av. Bulnes, 285, Santiago, Chile, hugo.rivera@conaf.cl
}

\begin{abstract}
Resumo - A Floresta Ombrófila Mista é considerada uma das principais formações do bioma Mata Atlântica. Apesar de sua importância, existe uma carência de informações sobre a dinâmica de crescimento de espécies dessa tipologia florestal. O presente estudo foi desenvolvido com o objetivo de estimar o crescimento passado de espécies arbóreas de Floresta Ombrófila Mista. Foram realizadas avaliações dendrocronológicas não destrutivas nas espécies Araucaria angustifolia, Ocotea porosa, Ilex paraguariensis, Cedrela fissilis, Ocotea puberula e Ocotea pulchella. $\mathrm{O}$ crescimento periódico médio em diâmetro até 2006 foi de aproximadamente $0,5 \mathrm{~cm}^{2} \mathrm{no}^{-1}$, para A. angustifolia, C. fissilis, O. porosa e O. pulchella. O. puberula e I. paraguariensis apresentaram crescimento periódico médio em diâmetro até 2006 igual ou superior a $0,9 \mathrm{~cm}$ ano ${ }^{-1}$. Foi observado uma aceleração do crescimento médio a partir das primeiras décadas do século passado. Sugere-se que esse padrão de crescimento seja consequência de variações climáticas locais ao longo desse período.
\end{abstract}

Termos para indexação: Dendrocronologia, crescimento diamétrico, floresta nativa, Araucaria angustifolia.

\section{Growth acceleration of subtropical tree species through 90 years in an Araucaria Forest}

\begin{abstract}
Araucaria Forest is considered one of the most important forest formations of Atlantic Forest Biome. Regarding its importance, there is a lack of information about growth dynamics of its forest species. The present study aimed at to estimate the past growth of selected tree species of Araucaria Forest. Dendrochronological analysis was carried out on non-destructive samples of Araucaria angustifolia, Ocotea porosa, Ilex paraguariensis, Cedrela fissilis, Ocotea puberula and Ocotea pulchella. The average periodic diameter increment until 2006 was approximately $0.5 \mathrm{~cm}_{\text {year }}{ }^{-1}$, for A. angustifolia, C. fissilis, O. porosa and O. pulchella. O. puberula and $I$. paraguariensis presented average periodic diameter increment until 2006 of $0.9 \mathrm{~cm}$ year ${ }^{-1}$ or greater. The growth rhythm has accelerated since the first decades of last century. It is suggested that this different growth pattern is a consequence of climatic changes throughout the growing period.
\end{abstract}

Index terms: Dendrochronology, diametric growth, native Forest, Araucaria angustifolia

\section{Introdução}

Com uma arquitetura de copas única no dossel superior de uma floresta, a Araucaria angustifolia (Bertol.) Ktze. caracteriza a Floresta Ombrófila Mista (FOM) ou Floresta com Araucária, que é uma mistura de floras com origens distintas: temperada (austrobrasileira) e tropical (afro-brasileira). Ocupa as porções planálticas em altitude média de $800 \mathrm{~mm}$ a $1200 \mathrm{~m}$, com chuvas bem distribuídas durante o ano, sendo fortemente influenciada pelas baixas temperaturas e pela ocorrência regular de geadas. De acordo com Leite \&
Klein (1990), a área ocupada por este tipo florestal antes do desmatamento era de cerca de $175 \mathrm{mil} \mathrm{km}{ }^{2}$, ocorrendo principalmente na região Sul do Brasil, com algumas áreas ocasionais nos estados de São Paulo, Minas Gerais e Rio de Janeiro. A intensidade da exploração madeireira, desmatamentos e queimadas, substituição da vegetação por pastagens, agricultura, reflorestamentos homogêneos com espécies exóticas e a ampliação das zonas urbanas no sul do Brasil, iniciados nos primeiros anos do século 20, provocaram uma dramática redução da área das florestas originais na região (Medeiros et al., 2005). Hoje, estima-se que os remanescentes de 
Floresta Ombrófila Mista, nos estágios primários ou mesmo avançados, não perfazem mais de $0,7 \%$ da área original (Brasil, 2002).

Sendo a araucária a principal espécie da FOM, não só pela dominância fitossociológica em relação às demais espécies arbóreas, mas também pelo valor econômico que possui, sua exploração intensificou-se em meados da década de 1930, afetando diretamente outras espécies que se desenvolvem com ela associadas, como Ocotea porosa (Nees \& C. Mart. ) Barroso, Ocotea puberula (Rich.) Nees, Ocotea puchella (Nees) Mez, Ilex paraguariensis A. St.-Hil. e Cedrela fissilis Vell. Nas áreas remanescentes desse tipo florestal encontramse as matrizes que poderão recolonizar naturalmente ou fornecer propágulos para plantios de recuperação de áreas degradadas. Uma forma de conservar os remanescentes da Floresta com Araucária é estimular o seu manejo em bases sustentáveis (Sanquetta, 1999). Segundo Medeiros et al. (2005), a instalação de Unidades de Conservação também facilitaria a preservação das áreas remanescentes. No entanto, apesar do grande esforço com restrições legais ao uso desses remanescentes, as ações ainda são insuficientes para garantir sua recuperação e conservação.

A dinâmica de crescimento de uma floresta apresenta alta complexidade, heterogeneidade e lentidão nesses ecossistemas, e a sua compreensão é muito importante para a elaboração de um plano de manejo voltado à conservação das espécies (Schaaf et al, 2005). Nesse contexto, os estudos com anéis de crescimento complementam as informações obtidas pelo monitoramento de parcelas permanentes, conferindo maior agilidade na recuperação de informações de crescimento das árvores por séries históricas longas.

Estudos com anéis de crescimento são muito utilizados em regiões de clima temperado para obter informações sobre crescimento, mudanças ambientais e embasar planos de manejo florestal (Cherubini et al., 2003; Spiecker, 2002). Estudos dendrocronológicos com espécies tropicais começaram no início do século passado, avançando muito lentamente. Atualmente, já existe vasta literatura disponível com informações sobre influências ambientais no crescimento de espécies tropicais e subtropicais (Worbes, 2002). No entanto, ainda existe uma grande lacuna no conhecimento sobre o crescimento e sua resposta a diferentes condições ambientais, em função da diversidade de espécies arbóreas nessas regiões. Estudos dendrocronológicos com espécies da FOM têm revelado resultados importantes para a recuperação de informações de crescimento passado (Mattos et al., 2007a, 2007b, Scheeren, et al. 2002, Seitz \& Kanninen, 1989, Oliveira, 2007).

Este estudo teve por objetivo recuperar informações sobre o crescimento passado de seis espécies nativas da Floresta Ombrófila Mista, presentes na RFEE, a partir da análise dos anéis de crescimento.

\section{Material e métodos}

O material estudado provém da Reserva Florestal Embrapa/Epagri (RFEE), localizada no Município de Caçador, Santa Catarina, Brasil.

Segundo Schäffer \& Prochnow (2002), o Estado de Santa Catarina apresenta cerca de $17 \%$ da sua cobertura vegetal original, sendo que $280 \mathrm{mil}$ ha são de florestas primárias e 1.382.000 ha de florestas secundárias, como a Reserva Florestal Embrapa/Epagri (RFEE), que se encontra em estado de regeneração avançado. Segundo Rosot et al. (2007), a Reserva é um dos maiores fragmentos contínuos da FOM, representando uma comunidade onde é possível encontrar, além da fauna, característica da tipologia florestal, todos os estratos e espécies de grande valor econômico e ecológico como a imbuia, canelas, cedros e, de forma a marcar fortemente a paisagem, uma população de pinheiros de grandes dimensões.

De acordo com a classificação de Köppen, o clima local é do tipo $\mathrm{Cfb}$, mesotérmico, com verões frescos e sem estação seca definida. A área de estudo possui temperatura média anual entre $16^{\circ} \mathrm{C}$ e $17^{\circ} \mathrm{C}$, sendo que a máxima e a mínima média anual não ultrapassam $25^{\circ} \mathrm{C}$ e $11^{\circ} \mathrm{C}$, respectivamente. A precipitação anual varia entre $1.300 \mathrm{~mm}$ e $1.500 \mathrm{~mm}$ (Pandolfo et al., 2002).

As amostras de madeira foram coletadas de árvores com diâmetro superior a $10 \mathrm{~cm}$, a $1,30 \mathrm{~m}$ de altura (diâmetro à altura do peito - DAP), representantes de diferentes classes diamétricas (Tabela 1), extraídas do tronco à altura do DAP, pelo método não destrutivo, com trado de incremento. Após secas à temperatura ambiente, as amostras foram lixadas, para facilitar a visualização dos anéis de crescimento. A coleta e preparo dessas amostras foram descritos por Rivera (2007).

As principais características ecológicas e dos limites dos anéis de crescimento, para as seis espécies, estão descritas na Tabela 2. 
Tabela 1. Número de amostras coletadas por espécie e amplitude de diâmetros das árvores amostradas.

\begin{tabular}{lcc}
\hline Espécie & Número de amostras & Diâmetro (cm) \\
\hline Araucaria angustifolia (Araucareaceae) & 9 & $27-115$ \\
Ilex paraguariensis (Aquifoliaceae) & 8 & $11-60$ \\
Ocotea porosa (Lauraceae) & 10 & $33-93$ \\
Ocotea puchella (Lauraceae) & 7 & $10-52$ \\
Ocotea puberula (Lauraceae) & 8 & $10-60$ \\
Cedrela fissilis (Meliaceae) & 6 & $10-71$ \\
\hline
\end{tabular}

Tabela 2. Características ecológicas e dos anéis de crescimento das espécies estudadas.

\begin{tabular}{|c|c|c|}
\hline Espécie & Características gerais & Características dos anéis de crescimento \\
\hline A. angustifolia & $\begin{array}{l}\text { Espécie heliófila, colonizadora de campos, formando } \\
\text { agrupamentos esparsos no início, tornando-se mais densos } \\
\text { até construir os capões de pinhais (Rondon Neto, 2003). } \\
\text { Pode apresentar comportamento de espécie pioneira, ou } \\
\text { de espécie clímax (Soares, 1979). }\end{array}$ & $\begin{array}{l}\text { Anéis definidos pela diferença de lenho tardio e } \\
\text { inicial, demarcados pelo espessamento das paredes } \\
\text { dos traqueídeos (Rondon Neto, 2003). }\end{array}$ \\
\hline I. paraguariensis & $\begin{array}{l}\text { Espécie esciófila. Cresce preferencialmente em associação } \\
\text { com a araucária. É considerada espécie climácica, aceita } \\
\text { bem sombreamento em qualquer idade, tolerando mais } \\
\text { luz na fase adulta (Carvalho, 1994). }\end{array}$ & $\begin{array}{l}\text { Os anéis são delimitados por parênquima paratraqueal } \\
\text { escasso e difuso e em agregados, poros difusos, } \\
\text { dispersos e solitários, fibras com pontuações areoladas } \\
\text { e parede celular muito fina (Gonçalves, 2005). }\end{array}$ \\
\hline O. porosa & $\begin{array}{l}\text { Espécie esciófila, exigente em sombreamento na fase } \\
\text { juvenil. Espécie clímax, raramente comportando-se como } \\
\text { secundária, infiltrando-se em matas abertas e capoirões } \\
\text { (Carvalho, 1994). }\end{array}$ & $\begin{array}{l}\text { Os anéis são delimitados por uma linha tangencial de cor } \\
\text { mais escura, formada pelo espessamento e achatamento } \\
\text { das paredes das fibras (Rondon Neto, 2003). }\end{array}$ \\
\hline O. puchella & $\begin{array}{l}\text { Espécie esciófila de pequeno porte quando estabelecidas } \\
\text { em formações campestres, atingindo maior porte quando } \\
\text { no interior de florestas (Spathelf et al., 2000). A espécie } \\
\text { é considerada como secundária inicial, com tolerância à } \\
\text { luminosidade moderada (Carvalho, 1994). }\end{array}$ & $\begin{array}{l}\text { Os limites dos anéis são facilmente identificáveis, } \\
\text { sendo que o lenho inicial apresenta cor creme, com } \\
\text { poros de dimensões e distribuição uniformes, já o } \\
\text { lenho tardio apresenta cor pardo-escura, com maior } \\
\text { concentração de fibras (Spathelf et al., 2000). }\end{array}$ \\
\hline O puberula & $\begin{array}{l}\text { Espécie heliófila, comuns em capoeiras e capoeirões. } \\
\text { Secundária inicial, tolerante a sombremento leve a } \\
\text { moderado, na fase juvenil (Carvalho, 1994). }\end{array}$ & Semelhante à Ocotea porosa (Rondon Neto, 2003). \\
\hline C. fissilis & $\begin{array}{l}\text { Trata-se de uma espécie heliófila ou de luz difusa (Klein, } \\
\text { 1984). É caducifolia e se estabelece tanto em florestas } \\
\text { primárias quanto em secundárias. Comporta-se como } \\
\text { secundária inicial ou tardia, necessitando de luz plena na } \\
\text { fase adulta (Carvalho, 1994). }\end{array}$ & $\begin{array}{l}\text { Os anéis são delimitados por faixas de parênquima } \\
\text { axial marginal, sendo os anéis semiporosos (Iwasaki- } \\
\text { Marochi, 2007). }\end{array}$ \\
\hline
\end{tabular}

Os anéis de crescimento de cada amostra foram marcados e medidos, utilizando-se microscópio estereoscópico e mesa de mensuração, com precisão de 0,01 mm (TSAP, 1996).

Os incrementos médios foram processados separadamente por espécie, em retrospectiva, gerando incrementos médios anuais por classes diamétricas. Foram consideradas seis classes de DAP, sem casca, sendo elas: 0 a $20 \mathrm{~cm} ; 20,1 \mathrm{~cm}$ a $40 \mathrm{~cm} ; 40,1 \mathrm{~cm}$ a $60 \mathrm{~cm}$; $60,1 \mathrm{~cm}$ a $80 \mathrm{~cm} ; 80,1 \mathrm{~cm}$ a 100 e acima de $100,1 \mathrm{~cm}$.

Os incrementos médios anuais das árvores de cada espécie foram também processados de modo a agrupar os dados de incremento médio anual em diâmetro para cada espécie. A partir dessas médias anuais geradas para cada espécie, os dados foram processados em médias de décadas, considerando a década mais recente o intervalo entre 1997 e 2006, retrocedendo até a década entre 1917 e 1926.

Foi realizada a análise de variância para identificar se as diferenças entre médias eram significativas. Foi utilizado o teste Tukey a $1 \%$ de probabilidade, entre as médias das classes diamétricas e entre as médias decadais, para identificar as médias estatisticamente diferentes entre si.

Os incrementos médios anuais em diâmetro, incluindo-se todos os indivíduos em estudo, foram utilizados para ajustar uma equação de regressão. 


\section{Resultados}

O incremento periódico anual apresentou uma grande amplitude para as seis espécies estudadas, mas, em média, manteve-se constante nos últimos 20 anos, exceto em Cedrela fissilis, que apresentou uma elevação de mais de $20 \%$ no IPA para o mesmo período. As espécies com crescimento mais rápido foram $I$. paraguariensis e $O$. puberula, com IPA superior a $0,90 \mathrm{~cm}$. O. puchella foi a espécie com crescimento mais lento (Tabela 3 ).

O incremento médio, por classe diamétrica, incluindose os dados reais e os incrementos recuperados pela estimativa em retrospectiva, utilizando os valores do incremento anual de cada árvore estão apresentados, por espécie, na Figura 1. Em geral, para uma mesma espécie,
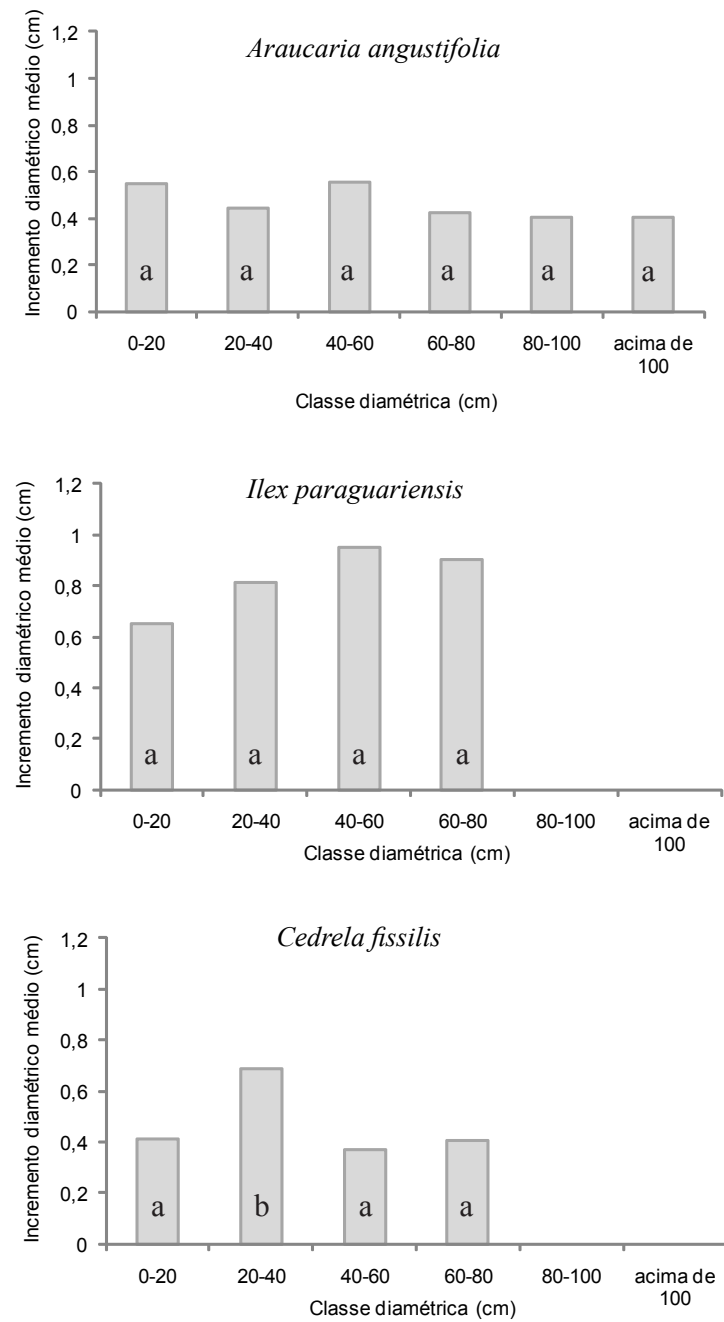

o crescimento em diâmetro é semelhante nas diferentes classes diamétricas consideradas.

Tabela 3. Incremento médio em diâmetro por espécie, com o incremento mínimo e máximo observados para a espécie no período de estudo, entre parênteses.

\begin{tabular}{lcc}
\hline Espécie & $\mathbf{I P A}_{\mathbf{2 0}}{ }^{(\mathbf{1})}(\mathbf{c m})$ & $\mathbf{I P A}_{\mathbf{1 0}}{ }^{(\mathbf{2})}(\mathbf{c m})$ \\
\hline Araucaria angustifolia & $\mathbf{0 , 5 0}(0,13-0,81)$ & $\mathbf{0 , 5 3}(0,16-0,95)$ \\
Ilex paraguariensis & $\mathbf{0 , 9 0}(0,29-1,37)$ & $\mathbf{0 , 9 4}(0,35-1,83)$ \\
Ocotea porosa & $\mathbf{0 , 5 7}(0,22-0,81)$ & $\mathbf{0 , 5 9}(0,13-0,99)$ \\
Ocotea puchella & $\mathbf{0 , 3 5}(0,21-0,52)$ & $\mathbf{0 , 3 2}(0,12-0,56)$ \\
Ocotea puberula & $\mathbf{0 , 9 8}(0,80-1,38)$ & $\mathbf{0 , 9 8}(0,63-1,33)$ \\
Cedrela fissilis & $\mathbf{0 , 5 7}(0,32-0,89)$ & $\mathbf{0 , 7 0}(0,37-1,36)$ \\
\hline
\end{tabular}

(1) Incremento periódico anual dos últimos 20 anos; ${ }^{(2)}$ Incremento periódico anual dos últimos 10 anos.
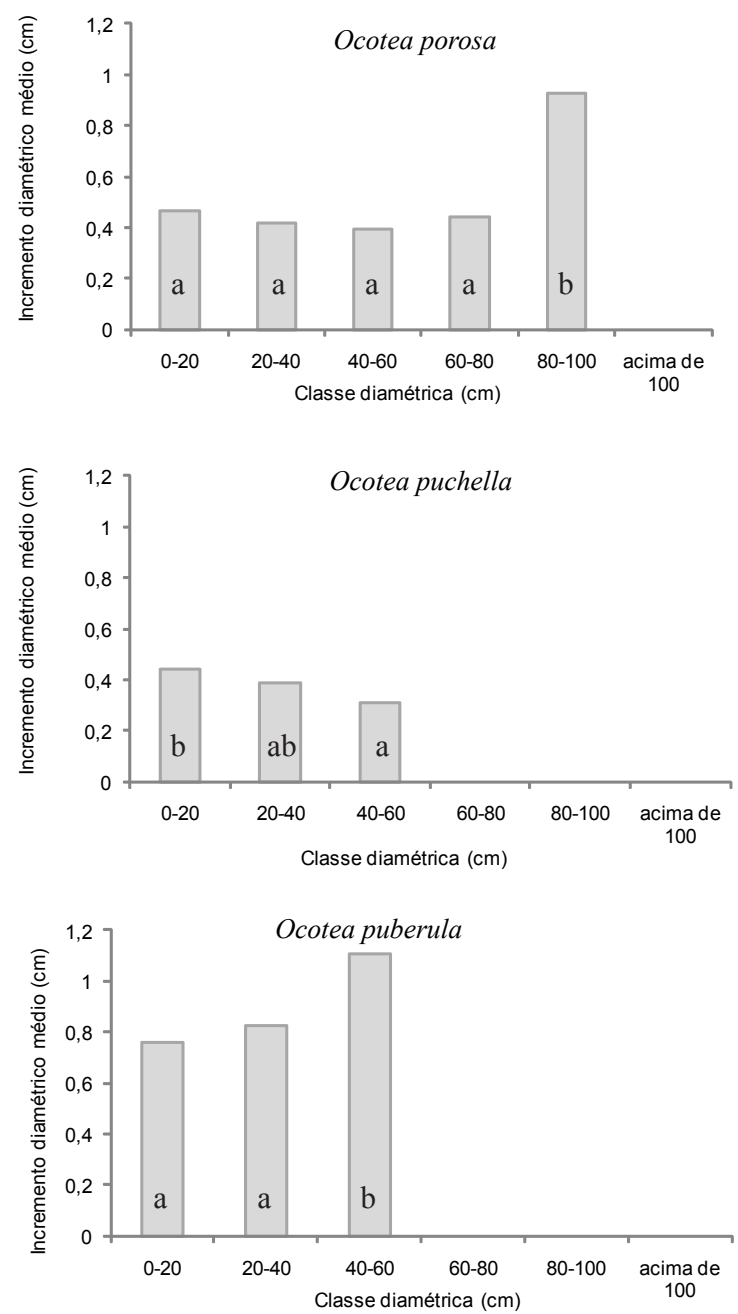

Figura 1. Incremento diamétrico médio, por classe diamétrica, para as espécies $A$. angustifolia, O. porosa, I. paraguariensis, $O$. puchella, $C$. fissilis e $O$. puberula. Barras com a mesma letra, para cada espécie, não apresentam diferenças significativas para incremento médio em diâmetro pelo teste Tukey a $1 \%$ de probabilidade. 
Para $O$. porosa foi observado incremento médio mais elevado apenas para a classe diamétrica maior $(80$ $\mathrm{cm}$ a $100 \mathrm{~cm}$ de diâmetro), praticamente o dobro do observado nas classes de diâmetro inferiores. Também pode-se destacar o crescimento mais elevado de $O$. puberula, para os indivíduos entre $40 \mathrm{~cm}$ e $60 \mathrm{~cm}$ de diâmetro e de Cedrela fissilis, entre $20 \mathrm{~cm}$ e $40 \mathrm{~cm}$ de diâmetro. $O$. pulchella apresentou diferença significativa de incremento médio anual entre a menor $(0,44 \mathrm{~cm})$ e a maior classe diamétrica $(0,31 \mathrm{~cm})$.

Podem-se destacar as espécies I. paraguariensis e Ocotea puberula, que mantiveram o incremento médio em todas as classes diamétricas superior a $0,60 \mathrm{~cm} \mathrm{ano}^{-1}$, sendo igualadas apenas por Cedrela fissilis, entre $20 \mathrm{~cm}$ e $40 \mathrm{~cm}$, e $O$. porosa, em diâmetros acima de $60 \mathrm{~cm}$.

As médias dos incrementos anuais foram agrupadas por décadas, para cada espécie, para melhor visualização da oscilação do crescimento ao longo dos últimos 90 anos. Foram observadas diferenças significativas entre períodos de crescimento anteriores a 1950, quando comparados as médias de crescimento médio da última década avaliada (1997-2006), para cinco espécies (Tabela 4).

Observa-se um período de crescimento mais lento nas décadas de 1917-1926 a 1927-1936 para as três espécies com séries mais longas (A. angustifolia, $C$. fissilis e $O$. porosa). A partir daí, ocorre oscilação em diferentes momentos para cada espécie, com uma tendência de aumento do incremento médio, até o período de 1997 2006. Cedrela fissilis e O. pulchella apresentaram picos de crescimento mais elevado, em 1947-1956 e 19671976 e na última década, que correspondeu à elevação do crescimento médio para praticamente todas as espécies, com exceção de $O$. pulchella (Tabela 4).

Tabela 4. Crescimento médio anual em diâmetro, em períodos decadais para seis espécies da FOM*.

\begin{tabular}{lllcclc}
\hline Décadas & A. angustifolia & O. porosa & O. puberula & O. pulchella & C. fissilis & I. paraguariensis \\
\hline $1917-1926$ & $0,23 \mathrm{~b}$ & $0,37 \mathrm{bcd}$ & - & - & $0,29 \mathrm{bcd}$ & - \\
$1927-1936$ & $0,26 \mathrm{~b}$ & $0,31 \mathrm{~d}$ & - & - & $0,15 \mathrm{~cd}$ & - \\
$1937-1946$ & $0,53 \mathrm{a}$ & $0,30 \mathrm{~d}$ & - & $0,36 \mathrm{~cd}$ & $0,11 \mathrm{~d}$ & - \\
$1947-1956$ & $0,47 \mathrm{ab}$ & $0,35 \mathrm{dc}$ & - & $0,60 \mathrm{a}$ & $0,54 \mathrm{ab}$ & - \\
$1957-1966$ & $0,45 \mathrm{ab}$ & $0,33 \mathrm{dc}$ & - & $0,53 \mathrm{abc}$ & $0,32 \mathrm{bcd}$ & $0,29 \mathrm{~b}$ \\
$1967-1976$ & $0,37 \mathrm{ab}$ & $0,38 \mathrm{bcd}$ & $0,35 \mathrm{~b}$ & $0,56 \mathrm{ab}$ & $0,51 \mathrm{ab}$ & $0,53 \mathrm{ab}$ \\
$1977-1986$ & $0,53 \mathrm{a}$ & $0,51 \mathrm{abc}$ & $0,73 \mathrm{a}$ & $0,39 \mathrm{bcd}$ & $0,33 \mathrm{bcd}$ & $0,62 \mathrm{ab}$ \\
$1987-1996$ & $0,47 \mathrm{ab}$ & $0,55 \mathrm{ab}$ & $0,89 \mathrm{a}$ & $0,37 \mathrm{~cd}$ & $0,44 \mathrm{abc}$ & $0,78 \mathrm{a}$ \\
$1997-2006$ & $0,53 \mathrm{a}$ & $0,59 \mathrm{a}$ & $0,98 \mathrm{a}$ & $0,32 \mathrm{~d}$ & $0,70 \mathrm{a}$ & $0,94 \mathrm{a}$
\end{tabular}

*Médias seguidas da mesma letra, para cada espécie, não apresentam diferenças significativas para incremento médio em diâmetro na década, pelo teste Tukey a $1 \%$ de probabilidade.

O crescimento interdecadal das espécies estudadas apresentou a mesma tendência de aumento, com exceção da $O$. pulchella. A inclinação da reta de regressão gerada a partir dos dados de incremento médio anual de todas as árvores em estudo indica que a diferença observada nas décadas se manifesta no aumento do incremento médio anual (Figura 2).

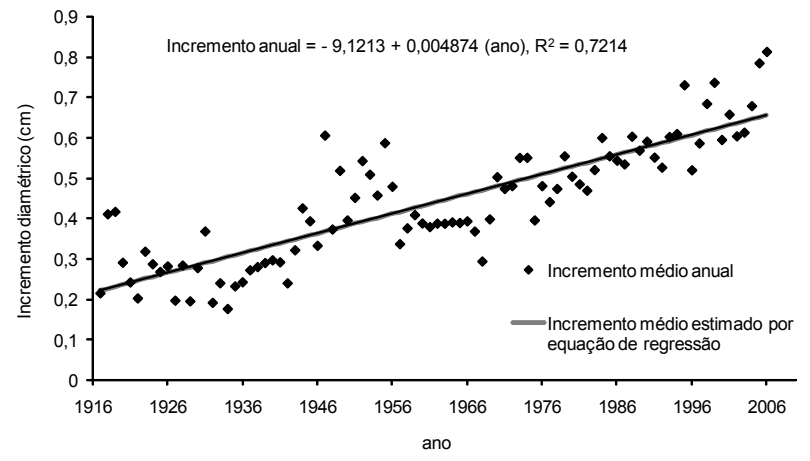

Figura 2. Média anual do crescimento em diâmetro das seis espécies estudadas e crescimento estimado pela equação de regressão. 


\section{Discussão}

Os valores de incremento médio obtidos para as espécies $O$. porosa, A. angustifolia e C. fissilis são semelhantes aos encontrados por Rondon Neto (2003) em estudo realizado em um remanescente de FOM Montana, em que foi observado incremento médio anual em diâmetro de $0,63 \mathrm{~cm}$ para $A$. angustifolia, $0,3 \mathrm{~cm}$ para $O$. porosa e $0,55 \mathrm{~cm}$ para $O$. puberula. Iwasaki-Marochi (2007) encontrou um IMA de $0,8 \mathrm{~cm}$ para $C$. fissilis em Campo Belo do Sul, SC, e na Floresta Nacional de São Francisco de Paula. Oliveira (2007) observou $0,74 \mathrm{~cm}$ de incremento médio anual em diâmetro para A. angustifolia. Spathelf et al. (2000) observaram crescimento entre 0,28 e 0,44 para $O$. pulchella na região da Serra Geral de Santa Maria, atribuindo o crescimento mais lento à competição com outras árvores.

No entanto, para a região de Caçador, SC, o incremento diamétrico médio anual de indivíduos de $O$. puberula, obtidos no presente estudo, foram superiores aos relatados por Rondon Neto (2003). O mesmo foi observado quando comparado com resultados obtidos por Schäaf et al. (2005), em São João do Triunfo, PR, para O. Porosa, A. angustifolia, I. paraguariensis, $O$. puberula e C. fissilis, e por Figueiredo et al. (2003), para $A$. angustifolia e $O$. porosa, também em São João do Triunfo, PR.

As diferenças de incremento médio em diâmetro observadas entre a Reserva Florestal Embrapa/Epagri, em Caçador, e outros remanescentes da Floresta com Araucária são, provavelmente, resultantes das diferenças de clima, solo, características de conservação dos remanescentes em que estão crescendo, além de variabilidade genética das populações naturais. Oliveira (2007) observou que déficit hídrico durante a estação de crescimento resultava em anéis mais estreitos em Araucaria angustifolia e, possivelmente, temperatura mais alta ao final da estação de crescimento influencia positivamente o crescimento do ano corrente ou do ano seguinte. Além disso, este autor observou uma correlação complexa entre comprimento do dia e temperatura influenciando diretamente o crescimento secundário de A. angustifolia.

Nota-se que, além das diferenças de crescimento entre locais, observaram-se diferenças de crescimento entre classes diamétricas, independentes do período de crescimento. Apesar de este resultado estar baseado em amostragem pequena, é um indicativo importante da necessidade de se adotarem técnicas de manejo distintas entre espécies ou grupos de espécies, identificando e favorecendo as classes diamétricas mais produtivas de cada espécie (Braz, 2010).

$\mathrm{O}$ incremento anual em diâmetro em árvores adultas tende a estabilizar ao longo do tempo, antes de atingir o declínio natural, sendo que cada indivíduo ou espécie reflete suas próximas condições e limitações de crescimento. Oscilações anuais de maior crescimento como consequência a anos com condições mais favoráveis são esperadas. No entanto, quando observamos o crescimento interdecadal médio das seis espécies (Tabela 4), com aumento do incremento anual em diâmetro, mesmo em árvores com grandes diâmetros (DAP) e com altura já estabilizada, indepentende de sua posição fitossociológica ou de suas características ecológicas (Tabela 2), podemos sugerir que o crescimento dessas plantas está respondendo diretamente ao ambiente, seja por variações nas condições climáticas ao longo do tempo ou por interferências antrópicas. Alguns estudos já apresentaram a relação do crescimento de árvores de espécies tropicais em escala interdecadal com o clima (Barichivich et al., 2009; Schongart et al., 2006).

$O$. pulchella foi a única espécie que não apresentou aumento do crescimento nas últimas duas décadas, o que sugere características de espécie de ciclo curto, com declínio natural do seu incremento médio em diâmetro.

O aumento do crescimento ao longo dos últimos 60 a 70 anos fica mais evidente quando trabalhamos as médias de crescimento anual de todas as árvores estudadas. Esse comportamento já foi relatado recentemente para Pinus longaeva, no noroeste dos Estados Unidos (Salzer et al., 2009), Abies cephalonica, em ilhas gregas (Koutavas, 2008), povoamentos de Fagus sylvatica, no nordeste da França (Bomtemps et al., 2010) e Abies alba na região dos Alpes, também na França (Chauchard et al., 2010). Em trabalho de reconstrução climática por anéis de crescimento na Província de Qianghai, na China, com Sabina przewalskii Kom., Zhu et al. (2008) observaram que o século 20 foi o mais quente, e a década de 1990 a mais quente, nos últimos 1.000 anos. A justificativa mais frequente nesses estudos, para esse comportamento, é o aumento do carbono, reflexo de mudanças climáticas globais. Sugere-se uma amostragem mais sistemática para confirmar esse comportamento e suas causas, semelhante ao sugerido por Koutavas (2008). 


\section{Conclusões}

Observa-se diferença no crescimento médio das classes diamétricas em Ocotea porosa, Cedrela fissilis, Ocotea puberula e Ocotea pulchella.

A aceleração do crescimento médio anual ao longo das últimas décadas sugere comportamento semelhante ao observado por outros autores, como consequência do aquecimento global ou aumento da concentração de carbono na atmosfera. No entanto, a confirmação dessa hipótese depende de estudos específicos sobre o tema.

\section{Referências}

BARICHIVICH, J.; SAUCHYN, D. J.; LARA, A. Climate signals in high elevation tree-rings from the semiarid Andes of northcentral Chile: Responses to regional and large-scale variability. Palaeogeography, Palaeoclimatology, Palaeoecology, v. 281, p. 320-330, 2009. doi:10.1016/j.palaeo.2007.10.033

BRAZ, E. M. Subsídios para o planejamento de manejo de florestas tropicais da Amazônia. 2010. 236 f. il. Tese (Doutorado em Engenharia Florestal) - Universidade Federal de Santa Maria, Santa Maria.

BOMTEMPS, J. D.; HERVE, J. C.; DHÔTE, J. F. Dominant radial and height growth reveal comparable historical variations for common beech in north-eastern France. Forest Ecology and Management, v. 259, p. 1455-1463, 2010. doi:10.1016/j. foreco.2010.01.019.

CARVALHO, P. E. R. Espécies florestais brasileiras: recomendações silviculturais, potencialidades e uso da madeira. Colombo: EMBRAPA-CNPF; Brasília, DF: EMBRAPA-SPI, 1994. $639 \mathrm{p}$

CHAUCHARD, S.; BEILHE, F.; DENIS, N.; CARCAILLET, C. An increase in the upper tree-limit of silver fir (Abies alba Mill.) in the Alps since the mid-20th century: A land-use change phenomenon. Forest Ecology and Management, v. 259, p. 14061415, 2010. doi:10.1016/j.foreco.2010.01.009

CHERUBINI, P.; GARTNER, B. L.; TOGNETTI, R.; BRAKER, O. U.; SCHOCH, W.; INNER, J. I. Indentification, measurement and interpretation of tree rings in woody species from Mediterranean climates. Biology Review, n. 78, p. 119-148, 2003. doi: $10.1017 / \mathrm{S} 1464793102006000$

FIGUEIREDO FILHO, A., HUBIE, S. do R.; SCHAAF, L. B.; FIGUEIREDO, D. J. de; SANQUETTA, C. R. Avaliação do incremento em diâmetro com o uso de cintas dendrométricas em algumas espécies de uma Floresta Ombrófila Mista localizada no Sul do Estado do Paraná. Ciências Exatas e Naturais, Guarapuava, v. 5, n. 1, p. 69-84, jan./jun. 2003.

GONÇALVES, T. A. P.; ZBOROWSKI, M. B.; SCHEELYBERT, R. Coleção de Referência Antracológica: Anatomia da madeira de Espécies de Anacardiaceae, Annonaceae, Aquifoliaceae, Apocynaceae, Araliaceae e Compositae de vários Biomas Brasileiros. In: CONGRESSO BRASILEIRO DA ASSOCIAÇÃO
BRASILEIRA DE ESTUDOS DO QUATERNÁRIO, 5., 2005, Guapari. Resumos expandidos. Boletim de Resumos. Guarapari, ES: Abequa, 2005. p. 171. 1 CD-ROM)

IWASAKI-MAROCHI, C. Anéis anuais de crescimento do cedro (Cedrela fissilis - Meliaceae) aplicados à avaliação da taxa de crescimento e dendroclimatologia. Curitiba, 2007. 120 f. Tese (Doutorado em Engenharia Florestal) - Setor de Ciências Agrárias, Universidade Federal do Paraná, Curitiba.

KLEIN, R. M. Meliaceae. In: REITZ, R. (Ed.). Flora ilustrada catarinense: as plantas meliáceas. Itajaí: Herbário Barbosa Rodrigues, 1984. p. 40-46.

KOUTAVAS, A. Late 20th century growth acceleration in greek firs (Abies cephalonica) from Cephalonia Island, Greece: A CO2 fertilization effect? Dendrochronologia, v. 26, p. 13-19, 2008. doi:10.1016/j.dendro.2007.06.001

LEITE, P. F.; KLEIN, R. M. Vegetação. In: IBGE. Diretoria de Geociencias. Rio de Janeiro, RJ. Geografia do Brasil: Região Sul. Rio de Janeiro: 1990. 420 p.

MATTOS, P. P. de; SANTOS, A. T. dos; OLIVEIRA, Y. M. M. de; ROSOT, M. A. D. Dendrocronologia de espécies da Floresta Ombrófila Mista do Município de Candói, PR. Pesquisa Florestal Brasileira, Colombo, n. 54, p. 153-156, jan./jun. 2007a. NOTA CIENTÍFICA.

MATTOS, P. P. de; SANTOS, A. T. dos; RIVERA, H.; OLIVEIRA, Y. M. M. de; ROSOT, M. A. D.; GARRASTAZU, M. C. Crescimento de Araucaria angustifolia na Reserva Florestal Embrapa/Epagri, Caçador, SC. Pesquisa Florestal Brasileira, Colombo, n. 55, p. 107-114, jul./dez. 2007b. NOTA CIENTÍFICA. MEDEIROS, J. D. de; SAVI, M.; BRITO, B. F. A. de. Seleção de áreas para criação de unidades de conservação na Floresta Ombrófila Mista. Biotemas, Florianópolis, v. 18, n. 2, p. 33-50, 2005.

BRASIL. Ministério do Meio Ambiente. Proposta do grupo de trabalho preservação e recuperação da Floresta Ombrófila Mista no Estado de Santa Catarina. Portaria Ministerial 49 de 06 de fevereiro de 2002, Brasília, Brasil, p. 77.

OLIVEIRA, J. M. Anéis de crescimento de Araucaria angustifolia (Bert.) O. Kuntze Araucaria angustifolia: bases de dendrocronologia em ecossistemas subtropicais montanos no Brasil. 2007. 139 f. Tese (Doutorado) - Universidade Federal do Rio Grande do SUL, Porto Alegre.

PANDOLFO, C.; BRAGA,H. J.; SILVA JÚNIOR, V. P.; MASSIGNAN, A. M.; PEREIRA, E. S.; THOMÉ, V. M. R. Atlas climatológico digital do Estado de Santa Catarina. Florianópolis: Epagri, 2002. 1 CD-ROM.

RIVERA, H. H. Ordenamento territorial de áreas florestais utilizando avaliação multicritério apoiada por geoprocessamento, fitossociologia e análise multivariada. Curitiba, 2007. 206 f. Dissertação (Mestrado em Engenharia Florestal) - Setor de Ciências Agrárias, Universidade Federal do Paraná, Curitiba. 
RONDON NETO, R. M. Análise do histórico silvicultural de quatro espécies de uma Floresta Ombrófila Mista com auxílio da dencronologia. Curitiba, 2003. 105f. Tese (Doutorado em Engenharia Florestal) - Setor de Ciências Agrárias, Universidade Federal do Paraná, Curitiba, 2003.

ROSOT, M. A. D.; OLIVEIRA, Y. M. M.; MATTOS, P. P.; GARRASTAZU, M. C.; SHIMIZU, J. Y. Monitoramento na Reserva Florestal da Embrapa/Epagri (RFEE) em Caçador, Santa Catarina. Colombo: Embrapa Florestas, 2007. (Embrapa Florestas. Documentos, 158).

SANQUETTA, C. R. Arausis: Sistema de simulação para manejo sustentável de florestas de Araucária. Revista Floresta, Curitiba, v. 29, n. 1-2, p. 115-121, 1999.

SALZER, M. W.; HUGHES, M. K.; BUNN, A. G.; KIPFMUELLER, K. F. Recent unprecedented tree-ring growth in bristlecone pine at the highest elevations and possible causes. PNAS, v. 106, n. 48, p. 20348-20353, 2009. doi: 10.1073 pnas.0903029106

SCHAAF, L. B.; FIGUEIREDO FILHO, A.; SANQUETTA, C. R.; GALVÃO, F. Incremento Diamétrico e em Área Basal no período de 1979-2000 de Espécies Arbóreas de uma Floresta Ombrófila Mista no Sul do Paraná. Floresta, Curitiba, v. 35, n. 2 , p. 271-290, maio/ago. 2005.

SCHÄFFER, W. B.; PROCHNOW, M. (Org.). A Mata Atlântica e você: como preservar, recuperar e se beneficiar da mais ameaçada floresta brasileira. Brasília: APREMAVI, 2002. 156 p.

SCHEEREN, L. W.; FLEIG, F. D.; SCHNEIDER, P. R.; FINGER, C. A. G. Crescimento de Canela-Lageana, Ocotea pulchella Nees et Mart. ex Nees, na depressão central do estado do Rio Grande do Sul. Ciência Florestal, Santa Maria, v. 13, n. 1, p. 2003. Ciência Florestal, Santa Maria, v. 13, n. 1, p. 137-144, jun. 2003. Nota técnica.
SCHONGART, J.; ORTHMANN, B.; HENNENBERG, K. J.; POREMBSKI, S.; WORBES, M. Climate-growth relationships of tropical tree species in West Africa and their potential for climate reconstruction. Global Change Biology, v. 12, p. 1139-1150, 2006.

SEITZ, R. A.; KANNINEN, M. Tree rings anlysis of Araucaria angustifolia in Southern Brazil: preliminary results. IAWA Bull, v. 10, n. 2, p. 170-174, 1989.

SOARES, R. V. Considerações sobre a regeneração natural da Araucaria angustifolia. Floresta, Curitiba, v. 10, n. 2, p. 12-18, 1979.

SPATHELF, P.; FLEIG, F. D.; VACCARO, S.; TONINI, H.; ESBER, L. M. Análise dendroecologica de Ootea pulchella de Nees et Mart. Ex nees (Canela-lageada) na Serra geral de Santa Maria, RS, Brasil. Ciência Florestal, Santa Maria, v.10, n.1, p. 95-108, 2000.

SPIECKER, H. Tree rings and forest management in Europe. Dendrochronologia, v. 20, n./1-2, p. 191-202, 2002.

WORBES, M. One hundred years of tree-ring research in the tropics - a brief history and an outlook to future challenges.

Dendrochronologia, v. 20, n. 1-2, p. 217-231, 2002.

ZHU, H. F.; ZHENG Y. H.; SHAO X. M.; LIU X. H.; XU Y.; LIANG E. Y. Millennial temperature reconstruction based on tree-ring widths of Qilian juniper from Wulan, Qinghai Province, China. Chinese Science Bulletin, v. 53, n. 24, p. 3914-3920, 2008.

Recebido em 15 de julho de 2010 e aprovado em 18 de outubro de 2010 